\title{
Utilisation d'un système de vote interactif pour l'enseignement de lecture critique d'article scientifique dans une faculté de médecine française
}

\author{
Using an electronic audience response system for teaching critical reading \\ of scientific articles in a french medical school
}

Parmi les technologies de l'information et de la communication pour l'enseignement (TICE), les systèmes de vote interactif (SVI) permettent aux étudiants de répondre instantanément, à l'aide de bô̂tiers connectés, à des interrogations simples sous forme de questions à choix multiples (QCM). Les résultats enregistrés peuvent servir à l'évaluation sommative des apprentissages mais surtout, l'affichage instantané des réponses permet également d'utiliser le SVI comme outil pédagogique interactif pour fournir un feedback aux étudiants au cours de l'enseignement. Ainsi, comme l'a montré une revue canadienne de 67 études réalisées sur l'enseignement de matières scientifiques au lycée ou à la faculté ${ }^{[1]}$, le SVI présente de nombreux avantages : amélioration de l'assiduité des étudiants, meilleure attention en classe, meilleure participation tout en respectant l'anonymat, interaction accrue (notamment entre les étudiants), discussion active des erreurs et apprentissage amélioré. Le SVI permet également une évaluation régulière (facilitant l'attention), formative (par exemple, autour de questions concernant de nouvelles notions) et comparative (chaque étudiant peut comparer ses réponses à celles du groupe). Les auteurs de cette revue indiquent toutefois des limites, qui sont de trois types : 1) limite technologique : les boîtiers perdus par les étudiants ou ne fonctionnant pas correctement sont une source de stress potentiellement délétère en conditions d'évaluation sommative ; 2) limite organisationnelle : le temps supplémentaire nécessaire à la préparation des questions, à l'installation et la distribution des boîtiers, aux réponses et aux discussions suscitées demande un investissement organisationnel important pour les équipes pédagogiques ; 3) limite humaine : les enseignants doivent d'abord apprendre à maîtriser l'outil et à adapter leur enseignement ; les discussions non maîtrisées peuvent être source de confusion ; de leur côté, les étudiants peuvent être stressés, frustrés ou distraits par le SVI. Certains peuvent mal accepter leur erreur (feedback négatif) lorsque le reste du groupe semble avoir acquis la bonne réponse. Une résistance au changement de mode d'apprentissage, se manifestant par un refus des efforts que demande l'enseignement interactif, peut également être observée. Enfin, la majorité des étudiants est réticente à l'utilisation des SVI pour l'évaluation sommative, pour l'évaluation de l'assiduité et lorsque leur anonymat n'est pas préservé ${ }^{[1]}$.

En France, dans le cadre des épreuves classantes nationales, l'épreuve de lecture critique d'article (LCA) évalue la capacité de l'étudiant à lire de façon critique et à analyser le contenu d'un article scientifique issu d'une revue médicale, en vue de son 
autoformation actuelle et future. À première vue, l'utilisation d'un SVI semble adaptée à l'enseignement de méthodologie appliquée à la LCA : d'une part, cet enseignement comporte souvent des concepts nouveaux, parfois complexes pour les étudiants; d'autre part, la présentation sous forme de QCM se rapproche des nouvelles méthodes docimologiques choisies pour les épreuves classantes nationales informatisées. Un groupe de chercheurs tchèques a décrit l'utilisation du SVI dans le cadre d'une formation à la LCA en troisième cycle ${ }^{[2]}$ mais, à notre connaissance, aucune étude ne rapporte l'utilisation de cet outil dans l'enseignement médical en France.

Nous avons réalisé une étude pour évaluer l'acceptabilité et l'intérêt pédagogique perçu par les étudiants et enseignants vis à vis de l'utilisation d'un SVI dans l'enseignement de méthodologie appliquée à la LCA à la faculté de médecine.

\section{Réalisation des enseignements}

Pour l'année universitaire 2012-2013, quatre ateliers utilisant un SVI (boîtiers de vote individuel avec six boutons de réponse) ont été intégrés à l'enseignement de méthodologie appliquée à la LCA, destiné aux 221 étudiants de troisième année à la Faculté de médecine de Montpellier-Nîmes. Le SVI a fonctionné dans l'ensemble des ateliers avec toutefois une aide fréquente des services techniques (notamment lors des premiers ateliers) et un délai d'une dizaine de minutes pour la distribution nominative des boîtiers. Le SVI était d'abord utilisé en début de chaque séance en vue d'une évaluation sommative des prérequis comptant pour le contrôle continu. Cependant, des erreurs d'enregistrement des notes dans quelques ateliers et les conditions d'utilisation en classe surchargée (plus de trente étudiants par atelier), favorables aux échanges de réponse entre étudiants, ont compromis l'utilisation prévue du système pour le contrôle continu. Le SVI a été également utilisé pour une évaluation formative lors de questions et de discussions autour de nouvelles notions ou d'exercices d'application (par exemple, au sujet des courbes de survie). À la fin du semestre, les étudiants et les neufs enseignants ont été invités à répondre à un questionnaire en ligne pour déterminer l'acceptabilité de l'utilisation du SVI et l'intérêt pédagogique perçu.

\section{Opinion des étudiants}

Au total, 115 étudiants (52\%) ont répondu au questionnaire. Parmi eux, $92 \%$ avaient été assidus à tous les ateliers, $76 \%$ étaient satisfaits ou très satisfaits de l'utilisation du SVI, $67 \%$ exprimaient un intérêt accru pour la matière enseignée et $55 \%$ étaient favorables à son déploiement dans d'autres unités d'enseignement. Comparé à un enseignement traditionnel, la majorité des étudiants jugeaient le SVI plus motivant $(78 \%)$, facilitant davantage l'attention (81\%), l'interactivité (76\%) et l'apprentissage $(70 \%)$. Les limites indiquées en commentaire libre concernaient principalement les problèmes techniques et les conditions d'utilisation en classe surchargée. Quelques commentaires soulignaient le caractère stressant de l'utilisation de la technique pour le contrôle continu, d'autres témoignaient d'une opposition au changement d'outil pédagogique et d'autres soulignaient le manque d'exploitation de l'outil par l'enseignant.

\section{Opinion des enseignants}

Les neuf enseignants se sont dits suffisamment formés et globalement satisfaits de l'utilisation du SVI, soulignant le caractère attractif ludique pour les étudiants, l'attention et l'interactivité accrues et l'intérêt du feedback de l'ensemble des étudiants. Huit sur neuf se sont sentis à l'aise avec l'outil et l'ont jugé utilisable sans trop de difficultés techniques. Les enseignants notaient toutefois la lourdeur logistique, le temps accru de gestion, de préparation et d'adaptation des contenus, et les difficultés d'utilisation en contrôle continu, qu'ils jugeaient néanmoins pas rédhibitoires.

\section{Discussion et conclusion}

En dépit de ses limites (questionnaires non validés, représentativité incertaine, absence de groupe de 
comparaison), notre étude montre que les étudiants et enseignants en LCA ont été satisfaits de l'utilisation d'un SVI : l'acceptabilité était bonne et la perception de l'intérêt pédagogique était positive. Les limites exprimées concernaient principalement les difficultés techniques rencontrées lors de la mise en place du SVI et témoignent d'un avis plutôt négatif sur son utilisation pour un contrôle continu. Les contraintes techniques et logistiques ne semblent pas représenter des facteurs limitants majeurs. De plus, elles diminuent progressivement avec l'utilisation répétée de la technique et elles sont moindres lors de l'utilisation de boîtiers non nominatifs. Ainsi, il nous apparait que le SVI a vocation à être utilisé plutôt pour une évaluation formative que sommative. Cette expérimentation, réalisée lors de l'année universitaire 20122013 à la faculté de Montpellier-Nîmes, a été poursuivie sans difficulté les deux années suivantes dans le même cadre, à l'exception de l'évaluation sommative abandonnée pour les raisons évoquées plus haut.

D'autres disciplines médicales pourraient bénéficier de l'utilisation d'un SVI comme outil pédagogique. Les précédents travaux sur le SVI permettent de donner quelques conseils aux enseignants désireux de l'utiliser. En termes de fréquence d'utilisation au cours de l'enseignement, il est suggéré qu'une utilisation du SVI toutes les 20 minutes environ entraine des effets bénéfiques significatifs sur le déficit d'attention des étudiants ${ }^{[1]}$. Le SVI est aussi particulièrement adapté au développement de l'enseignement par les pairs (peer instruction). Cette technique pédagogique peut se résumer ainsi : l'enseignant pose une question aux étudiants concernant la compréhension d'un concept puis leur présente les résultats du vote sans donner la réponse ; les étudiants sont ensuite amenés à discuter ces résultats entre eux et sont incités à convaincre leurs pairs en expliquant leur raisonnement ; un second vote est proposé, la réponse est donnée et poursuivie par une discussion générale accompagnée des explications de l'enseignant. Cette méthode a montré des effets positifs sur l'apprentissage et une amélioration des performances des étudiants aux examens ${ }^{[3]}$. En effet, pour certains étudiants, l'apprentissage est plus efficace lorsqu'ils reçoivent des explications exprimées par leurs pairs « dans leur langage ${ }^{[1]}$. Un autre élément important réside dans la rédaction des questions proposées au vote. Les questions les plus efficientes sont celles qui concernent un objectif pédagogique spécifique, qui suscitent un large éventail de réponses, qui font prendre conscience aux étudiants d'opinions autres que les leurs et qui explorent des ambiguïtés, des confusions ou des nouveaux contextes ${ }^{[1]}$.

S'il a été montré que les classes bénéficiant du SVI obtenaient de meilleurs résultats que les classes d'enseignement classique, son impact réel sur l'apprentissage est discuté et nécessite d'être encore évalué $^{[1,4]}$. Toutefois, comme les autres TICE, le SVI semble améliorer les comportements d'apprentissage ${ }^{[5]}$. Il faut donc poursuivre les études pour mieux évaluer l'impact du SVI dans l'enseignement des sciences médicales.

Les auteurs remercient Sophie Bastide, Pierre Dujols, Paul Landais, Nicolas Nagot, Nicolas Molinari, Thibault Mura, Marie-Christine Picot, Roxane Schaub et Fabienne Seguret pour leur contribution à la réalisation de cette étude.

\section{Jean-Luc FAILLIE ${ }^{1,2}$ Philippe LAMBERT ${ }^{3}$ Dorine NEVEU ${ }^{4}$ Pascale FABBRO-PERAY ${ }^{5}$}

${ }^{1}$ Département de Pharmacologie Médicale et Toxicologie, CHRU Montpellier, France mailto : jl-faillie@ chu-montpellier.fr

2 Département de Pharmacoépidémiologie, INSERM U 1027, Faculté de Médecine, Université Paul Sabatier, Toulouse, France

${ }^{3}$ Département de Médecine Générale, Université de Montpellier, France

${ }^{4}$ Université de Montpellier, INSERM U 1058, CHRU Montpellier, France

${ }^{5}$ Laboratoire de Biostatistique, Épidémiologie, Santé Publique et Informatique Médicale, CHU Nîmes, France

\section{Références}

1. Kay RH, LeSage A. Examining the benefits and challenges of using audience response systems: A 
review of the literature. Computers \& Education 2009;53:819-27.

2. Mohr P, Hoschl C, Volavka J. Teaching critical appraisal of articles on psychopharmacology. Acad Psychiatry 2012;36:114-7.

3. Crouch $\mathrm{CH}$, Mazur E. Peer instruction: ten years of experience and results. Am J Phys 2001;69:970-77.
4. Lantz ME. The use of "Clickers" in the classroom: Teaching innovation or merely an amusing novelty? Comput Hum Behav 2010;26:556-61.

5. Ministère de l'Education Nationale. Les TIC : éléments sur leurs usages et sur leurs effets. Note-évaluation. 2003 [On-line] Disponible sur : ftp://trf.education.gouv.fr/pub/edutel/dpd/noteeval/ne0301.pdf. 